\title{
Postprandial lipid responses to standard carbohydrates used to determine glycaemic index values
}

\author{
Sonia Vega-López ${ }^{1 *}$, Lynne M. Ausman ${ }^{2}$, Nirupa R. Matthan ${ }^{2}$ and Alice H. Lichtenstein ${ }^{2}$ \\ ${ }^{1}$ Healthy Lifestyles Research Center, School of Nutrition and Health Promotion, Arizona State University, \\ 500 North 3rd Street, Phoenix AZ 85004, USA \\ ${ }^{2}$ Cardiovascular Nutrition Laboratory, Jean Mayer USDA Human Nutrition Research Center on Aging at \\ Tufts University, Boston, MA 02111, USA
}

(Submitted 3 August 2012 - Final revision received 12 February 2013 - Accepted 13 March 2013 - First published online 9 May 2013)

\begin{abstract}
Prior studies assessing the metabolic effects of different types of carbohydrates have focused on their glycaemic response. However, the response of postprandial cardiometabolic risk indicators has not been considered in these studies. The present study assessed postprandial lipid responses to two forms of carbohydrates used as reference foods for glycaemic index determinations, white bread (50 g available carbohydrate) and glucose $(50 \mathrm{~g})$, under controlled conditions and with intra-individual replicate determinations. A total of twenty adults (20-70 years) underwent two cycles of challenges with each pair of reference foods (four challenges/person), administered in a random order on separate days under standard conditions. Serum lipids (total cholesterol, LDL-cholesterol, HDL-cholesterol, TAG and NEFA), glucose and insulin were monitored for $5 \mathrm{~h}$ post-ingestion. Oral glucose resulted in greater glycaemic and insulinaemic responses than white bread for the first $90 \mathrm{~min}$ and a greater subsequent decline after $120 \mathrm{~min}(P=0 \cdot 0001)$. The initial decline in serum NEFA concentrations was greater after the oral glucose than after the white bread challenge, as was the rebound after $150 \mathrm{~min}(P=0 \cdot 001)$. Nevertheless, the type of carbohydrate had no significant effect on postprandial total cholesterol, LDL-cholesterol and HDL-cholesterol concentrations. Following an initial modest rise in TAG concentrations in response to both challenges, the values dropped below the fasting values for oral glucose but not for the white bread challenge. These data suggest that the type of carbohydrate used to determine the glycaemic index, bread or glucose, has little or modest effects on postprandial plasma cholesterol concentrations. Differences in TAG and NEFA concentrations over the $5 \mathrm{~h}$ time period were modest, and their clinical relevance is unclear.
\end{abstract}

\section{Key words: Cholesterol: TAG: NEFA: Postprandial responses: Carbohydrate challenges}

In light of the increased healthcare burden associated with the increasing rates of the metabolic syndrome worldwide and its metabolic sequelae, efforts have been focused on assessing the glycaemic response to diets containing different types of carbohydrates with the aim of providing dietary guidance to minimise postprandial insulin and glucose excursions ${ }^{(1)}$. Over the years, different classification systems have been developed to characterise blood glucose excursions in response to carbohydrate-rich foods, including total amount of carbohydrate (carb-counting), simple relative to complex carbohydrate, fibre-rich relative to fibre-poor carbohydrate, unrefined relative to refined carbohydrate, and most recently glycaemic index and glycaemic load ${ }^{(2,3)}$. Whereas most efforts have been focused on assessing the glycaemic and insulinaemic responses to diets containing different types of carbohydrates $^{(1,4)}$, less attention has been focused on the effect of dietary carbohydrate type on other cardiometabolic risk factors, particularly plasma lipoproteins and NEFA.
Prospective studies have demonstrated that consumption of diets with a higher proportion of simple than complex carbohydrates results in less favourable cardiometabolic profiles ${ }^{(5,6)}$ and that diets high in cereal fibre are associated with lower CVD risk ${ }^{(7)}$. Results from cross-sectional studies using nationally representative data have suggested that 'added sugars' adversely affect fasting lipoprotein profiles ${ }^{(8)}$. Early evidence from intervention studies conducted in the 1970s, 1980s and 1990s suggested that whereas high-carbohydrate/low-fat diets resulted in higher fasting and postprandial TAG concentrations compared with control diets in the absence of body weight change ${ }^{(9,10)}$, the isoenergetic replacement of complex carbohydrates with sucrose did not affect fasting plasma lipid concentrations ${ }^{(11)}$. Subsequent intervention studies that have focused on foods with high- and low-glycaemic index values have reported similar effects for fasting lipoprotein profiles, and only modest declines in fasting TAG concentrations in the absence of weight loss ${ }^{(1,12,13)}$. 
In contemporary settings, humans spend the majority of their time in the postprandial state rather than in the fasting state. The differential postprandial blood glucose responses to foods containing carbohydrates of diverse nature have been well documented since the late $1970 \mathrm{~s}^{(14-17)}$. However, there is limited information about the effects of the acute intake of different types of dietary carbohydrates on postprandial serum lipid and lipoprotein concentrations. Potential differences have direct implications for treatment guidelines. The present study was conducted to assess the postprandial lipid responses to the acute consumption of two carbohydrate sources currently used as reference foods to determine glycaemic index values, white bread and glucose ${ }^{(3,18)}$, under controlled conditions and with intra-individual replicate determinations.

\section{Experimental methods \\ Study population}

Study participants ( $n$ 25, fifteen females and ten males; 20-70 years) were recruited from the Greater Boston area. Exclusion criteria were as follows: known chronic disease (diabetes, CVD, kidney, thyroid and liver diseases); untreated hypertension; irritable bowel syndrome or malabsorptive disorder; smoking; BMI $>35 \mathrm{~kg} / \mathrm{m}^{2}$; fasting glucose $\geq 7 \mathrm{mmol} / 1$; abnormal blood chemistry or cell blood count; pregnancy; breast-feeding; alcohol consumption $>7$ drinks/week; use of medications known to affect glucose metabolism (insulin, sulphonylureas, metformin, glucosidase inhibitors and thiazolidinedione insulin sensitisers); weight gain or loss $>7 \mathrm{~kg}$ within 6 months before enrolment; unwillingness to adhere to the study protocol. The present study was conducted according to the guidelines laid down in the Declaration of Helsinki, and all procedures involving human subjects were approved by the Human Investigation Review Committee of Tufts University/Tufts Medical Center. Written informed consent was obtained from all the study participants. The present trial is registered at clinicaltrials.gov as NCT 01023646, 30 November 2009.

\section{Study protocol}

Replicate determinations were conducted on each subject. This was accomplished by having each participant complete two sets of determinations. Each set, or pair of visits, was conducted no more than $7 \mathrm{~d}$ apart and was used to assess the glycaemic and postprandial lipid responses to the acute consumption of white bread and glucose, administered in a random order. A staff member of the metabolic research unit administered the carbohydrate challenges so that the investigators could remain blinded as to the order. Only one participant was withdrawn from the study. However, four participants did not complete the two repeat sets. The remaining twenty participants (nine males and eleven females) had complete datasets and were included in the analyses. A small portion of data addressing a different experimental question has been reported previously ${ }^{(19)}$.

\section{Carbohydrate challenge}

The participants were requested to maintain their habitual diet and physical activity patterns during the entire test period, and were asked to fast and refrain from engaging in vigorous physical activity $12 \mathrm{~h}$ before each study day. Just before a subject's first carbohydrate challenge, blood pressure, height, weight and waist and hip circumferences were measured using standardised procedures. Immediately thereafter, an intravenous indwelling catheter was placed in the forearm of the subject for blood-drawing purposes and a fasting blood sample was obtained. The test food, either the equivalent of $50 \mathrm{~g}$ of available carbohydrate in the form of commercial white bread or glucose (described later), was provided and the subject was requested to consume the carbohydrate within a period of $5 \mathrm{~min}$. Additional blood samples were obtained at 15, 30, 45, 60, 90, 120, 150, 180, 210, 240, 270 and 300 min thereafter. During the test period, the participants were required to remain in our metabolic research unit and restricted to sedentary activities in their rooms. The environment, provision of test foods and blood collection procedures were maintained as consistent as possible on all the study days. For each set of visits, under supervision, the participants consumed $500 \mathrm{ml}$ of a glucose solution $(100 \mathrm{~g} / \mathrm{l} ; 50 \mathrm{~g}$ carbohydrate) or $96 \mathrm{~g}$ of commercial white bread (Original White Bread; Pepperidge Farm, Inc.; $50 \mathrm{~g}$ of available carbohydrate) with $500 \mathrm{ml}$ water, in a random order. Water was available ad libitum throughout the test period. Each set of studies was repeated twice and the means reported and used for the statistical analysis.

\section{Biochemical measures}

Blood was allowed to clot at room temperature for $20 \mathrm{~min}$ and serum was separated by centrifugation at $1100 \mathrm{~g}$ at $4^{\circ} \mathrm{C}$ for $20 \mathrm{~min}$. Glucose was measured using an enzymatic method (Olympus America, Inc.). The CV for the glucose determinations was $2 \%$. Insulin was measured using a human insulin- specific RIA kit (Linco Research). The $\mathrm{CV}$ for the insulin determina tions was 5\%. Serum total cholesterol, LDL-cholesterol, HDL-cholesterol and TAG concentrations were measured on a Hitachi 911 automated analyser (Roche Diagnostics) using enzymatic reagents. Serum NEFA were measured on a Cobas Fara II clinical chemistry analyser (Roche Diagnostic Systems, Inc.) using an enzymatic procedure (Wako Chemicals, Inc.). The assays were standardised through the Lipid Standardization Program of the Centers for Disease Control and Prevention, Atlanta, GA.

\section{Statistical methods}

Data were analysed using SAS for Windows version 9.2 (SAS Institute). The mean of the values obtained from each set of tests was used for this analysis. TAG data were inverse-transformed to achieve normality before the statistical analysis. Descriptive statistics and graphs (PROC UNIVARIATE and PROC MEANS) were used to summarise the overall effects of tests and distributions of the outcome measures. PROC MIXED with the main effects of treatment and time with repeated measures for the subjects was carried out. When 
the interaction treatment $\times$ time was significant at $P<0.05$, a paired $t$ test was carried out at each time point with Bonferroni's correction for multiple comparisons. Data are presented as means and standard deviations. The analysis was conducted at the $0.05 \alpha$-level and corrected for the number of comparisons made. Glycaemic index values are traditionally calculated using the incremental AUC for serum glucose concentrations over a $2 \mathrm{~h}$ period $^{(18)}$. However, the aim of the present study was to characterise the pattern of glucose, insulin and lipid concentrations over the period $5 \mathrm{~h}$ after the acute ingestion of oral glucose or white bread, not to determine the glycaemic index values, per se.

\section{Results}

The mean age of the participants was 45 (SD 15) years (Table 1). For the most part, their mean blood pressure and fasting serum lipid values were within optimal or near optimal values according to the ATP III guidelines (blood pressure $<130 / 850 \mathrm{mmHg}$; total cholesterol $<5.2 \mathrm{mmol} / \mathrm{l}$; LDL-cholesterol $<3.4 \mathrm{mmol} / \mathrm{l}$; HDL-cholesterol $\geq 1.0 \mathrm{mmol} / \mathrm{l}$; TAG $<1.7 \mathrm{mmol} / \mathrm{l})^{(20)}$. The mean BMI of the subjects was in the overweight range.

The postprandial glucose, insulin and lipid responses to the oral glucose and white bread challenges are summarised as absolute concentrations in Table 2 and as changes from fasting concentrations in Fig. 1. As predicted, the oral glucose challenge resulted in a greater glycaemic response than the white bread challenge during the first $90 \mathrm{~min}$ of the test period, with significantly higher serum glucose concentrations up to $60 \mathrm{~min}(P=0 \cdot 0001)$. Somewhat unexpected, during the subsequent $2 \mathrm{~h}$ period, the oral glucose challenge elicited serum glucose concentrations that were lower than the fasting values and significantly lower than those elicited by the white bread challenge $(P=0 \cdot 0001)$. A different pattern for serum insulin concentrations was observed during this time period $(P=0.0001)$; the insulinaemic response was greater after the oral glucose than after the white bread challenge at 15 and 30 min but not at the subsequent time points.

Postprandial serum total cholesterol, LDL-cholesterol and HDL-cholesterol concentrations were not significantly different for the two carbohydrate challenges over the $5 \mathrm{~h}$ time period (Table 2; Fig. 1). In contrast, serum TAG concentrations diverged after $90 \mathrm{~min}$, after which the oral glucose challenge resulted in lower concentrations, with the difference reaching statistical significance at $120 \mathrm{~min}(P=0.0165)$. Both carbohydrate challenges caused a decline in NEFA concentrations during the early phase of the observational period, which rebounded during the later phase. The pattern of response was significantly different for the two carbohydrate sources, with the excursions being more modest for the white bread challenge than for the oral glucose challenge $(P=0.0006$; Table 2).

\section{Discussion}

Although atherogenesis was identified as a postprandial phenomenon over 30 years ago ${ }^{(21)}$, the role of postprandial lipids in cardiometabolic diseases was not fully recognised until more recently ${ }^{(22)}$. This is an important point because in contemporary society, humans spend the majority of their time in the postprandial state. Given that there are limited data about the effects of the acute intake of different types of carbohydrates on postprandial cardiometabolic risk factors, the present study was carried out to characterise the postprandial lipid responses to two types of carbohydrates commonly used as reference foods for glycaemic index determinations and also prevalent in the current food supply, white bread and glucose, under controlled conditions.

In the present study, during the first part of the post-challenge period, the glycaemic and insulinaemic responses were greater after the oral glucose challenge than after the bread challenge, similar to that reported previously in early studies conducted in the 1970 s for the same challenges ${ }^{(14,15)}$

Table 1. Baseline characteristics of the participants

(Mean values, standard deviations and ranges)

\begin{tabular}{|c|c|c|c|c|c|c|c|c|c|c|}
\hline \multirow[b]{2}{*}{ Variables } & \multicolumn{3}{|c|}{ All participants $(n 20)$} & \multicolumn{3}{|c|}{ Males $(n 9)$} & \multicolumn{3}{|c|}{ Females $(n 11)$} & \multirow[b]{2}{*}{$P^{*}$} \\
\hline & Mean & SD & Range & Mean & SD & Range & Mean & SD & Range & \\
\hline Age (years) & 45 & 15 & $23-70$ & 49 & 14 & $35-69$ & 42 & 16 & $23-70$ & 0.3451 \\
\hline Systolic blood pressure (mmHg) & 114 & 14 & $89-142$ & 119 & 12 & $101-137$ & 110 & 14 & $89-142$ & 0.1523 \\
\hline Diastolic blood pressure $(\mathrm{mmHg})$ & 72 & 6 & $60-81$ & 74 & 5 & $66-81$ & 70 & 7 & $60-80$ & 0.1937 \\
\hline BMI $\left(\mathrm{kg} / \mathrm{m}^{2}\right)$ & $26 \cdot 5$ & 3.7 & $20 \cdot 3-32.5$ & $26 \cdot 7$ & $2 \cdot 9$ & $22 \cdot 4-29 \cdot 5$ & $26 \cdot 3$ & 4.4 & $20 \cdot 3-32.5$ & 0.8006 \\
\hline Waist circumference $(\mathrm{cm})$ & 89 & 13 & $67-117$ & 96 & 11 & $80-117$ & 83 & 10 & $67-99$ & 0.0125 \\
\hline Hip circumference $(\mathrm{cm})$ & 103 & 9 & $83-119$ & 102 & 9 & $91-119$ & 104 & 10 & $83-116$ & 0.7597 \\
\hline Glucose $(\mathrm{mmol} / \mathrm{l}) \dagger$ & 4.98 & 0.41 & $4.22-5.55$ & 5.09 & 0.35 & $4.44-5.55$ & 4.90 & 0.45 & $4 \cdot 22-5 \cdot 50$ & 0.3183 \\
\hline \multicolumn{11}{|l|}{ Serum lipids $(\mathrm{mmol} / \mathrm{l}) \dagger$} \\
\hline TC & 4.80 & 0.74 & $3.49-6.62$ & $4 \cdot 80$ & 0.67 & $3 \cdot 85-5 \cdot 79$ & 4.80 & 0.83 & $3.49-6.62$ & 0.9928 \\
\hline LDL-C & 2.90 & 0.58 & $2 \cdot 15-4 \cdot 32$ & 3.08 & 0.49 & $2 \cdot 30-3.85$ & $2 \cdot 75$ & 0.63 & $2 \cdot 15-4 \cdot 32$ & 0.2182 \\
\hline HDL-C & 1.50 & 0.35 & $1 \cdot 07-2 \cdot 17$ & 1.26 & 0.14 & $1.07-1.45$ & $1 \cdot 70$ & 0.35 & $1 \cdot 11-2 \cdot 17$ & 0.0022 \\
\hline TAG $\ddagger$ & 0.90 & 0.44 & $0.45-2.09$ & 1.04 & 0.40 & $0.45-1.69$ & 0.79 & 0.45 & $0.50-2.09$ & 0.2235 \\
\hline TC:HDL-C & $3 \cdot 3$ & 0.7 & $2 \cdot 3-4.9$ & 3.8 & 0.6 & $2.9-4.9$ & 2.9 & 0.5 & $2 \cdot 3-3 \cdot 7$ & 0.0011 \\
\hline
\end{tabular}

TC, total cholesterol; LDL-C, LDL-cholesterol; HDL-C, HDL-cholesterol.

${ }^{*} P$ values correspond to the significance of pairwise comparisons (males $v$. females).

† All values were measured in the fasting state.

‡TAG data were not normally distributed and were inverse-transformed before the analysis. 
Table 2. Postprandial serum glucose, insulin and lipid responses to the standard oral glucose (Glu) and white bread (Brd) challenges $\ddagger$

(Mean values and standard deviations, $n 20$ )

\begin{tabular}{|c|c|c|c|c|c|c|c|c|c|c|c|c|c|c|c|c|c|c|c|c|c|c|c|c|c|c|c|}
\hline & \multicolumn{27}{|c|}{ Time (min) } \\
\hline & \multicolumn{2}{|c|}{0} & \multicolumn{2}{|c|}{15} & \multicolumn{2}{|c|}{30} & \multicolumn{2}{|c|}{45} & \multicolumn{2}{|c|}{60} & \multicolumn{2}{|c|}{90} & \multicolumn{2}{|c|}{120} & \multicolumn{2}{|c|}{150} & \multicolumn{2}{|c|}{180} & \multicolumn{2}{|c|}{210} & \multicolumn{2}{|c|}{240} & \multicolumn{2}{|c|}{270} & \multicolumn{2}{|c|}{300} & \multirow[b]{2}{*}{$P \S$ (treatment $\times$ time) } \\
\hline & Mean & SD & Mean & SD & Mean & SD & Mean & SD & Mean & SD & Mean & SD & Mean & SD & Mean & SD & Mean & SD & Mean & SD & Mean & SD & Mean & SD & Mean & SD & \\
\hline \multicolumn{28}{|c|}{ Glucose $(\mathrm{mmol} / \mathrm{l})$} \\
\hline Glu & 4.80 & 0.41 & $6 \cdot 13^{\star}$ & 0.75 & $7.54^{*}$ & 0.82 & $7.78^{\star}$ & $1 \cdot 24$ & $7 \cdot 24^{\star}$ & 1.53 & 5.72 & 1.43 & 4.81 & $1 \cdot 27$ & $4 \cdot 20^{*}$ & 0.81 & $4 \cdot 11^{\star}$ & 0.51 & $4 \cdot 17^{*}$ & 0.44 & $4 \cdot 33$ & 0.37 & 4.49 & 0.33 & 4.63 & 0.31 & 0.0001 \\
\hline Brd & 4.78 & 0.34 & 4.81 & 0.37 & 5.91 & 0.92 & 6.47 & 0.84 & $6 \cdot 26$ & 0.90 & 5.48 & 0.84 & 5.05 & 0.93 & 4.85 & 0.89 & 4.79 & 0.72 & 4.72 & 0.51 & 4.55 & 0.34 & 4.51 & 0.34 & 4.58 & 0.32 & \\
\hline \multicolumn{28}{|c|}{ Insulin (pmo//) } \\
\hline Glu & 9 & 4 & $30^{*}$ & 13 & $52^{*}$ & 27 & 54 & 32 & 48 & 27 & 28 & 16 & 16 & 11 & $10 \dagger$ & 4 & $8 \dagger$ & 3 & 7 & 2 & 7 & 2 & 7 & 2 & 7 & 3 & 0.0001 \\
\hline Brd & 9 & 3 & 10 & 4 & 30 & 19 & 42 & 20 & 45 & 23 & 32 & 17 & 20 & 7 & 13 & 6 & 11 & 4 & 9 & 3 & 8 & 3 & 7 & 2 & 7 & 2 & \\
\hline \multicolumn{28}{|c|}{ TC (mmol/l) } \\
\hline Glu & 4.59 & 0.71 & 4.67 & 0.77 & 4.57 & 0.72 & 4.48 & 0.77 & 4.49 & 0.73 & 4.50 & 0.72 & 4.50 & 0.70 & 4.46 & 0.68 & 4.50 & 0.69 & 4.46 & 0.64 & 4.52 & 0.69 & 4.52 & 0.69 & 4.58 & 0.64 & 0.231 \\
\hline Brd & 4.60 & 0.58 & 4.69 & 0.66 & 4.57 & 0.62 & 4.54 & 0.66 & 4.46 & 0.59 & 4.55 & 0.62 & 4.57 & 0.60 & 4.48 & 0.60 & 4.50 & 0.57 & 4.48 & 0.58 & 4.52 & 0.65 & 4.55 & 0.67 & 4.58 & 0.65 & \\
\hline \multicolumn{28}{|c|}{ LDL-C (mmol/l) } \\
\hline Glu & 2.79 & 0.64 & 2.84 & 0.67 & 2.76 & 0.65 & 2.69 & 0.66 & 2.68 & 0.64 & 2.72 & 0.63 & 2.72 & 0.62 & 2.71 & 0.61 & 2.72 & 0.62 & 2.71 & 0.61 & 2.75 & 0.64 & 2.73 & 0.63 & 2.76 & 0.59 & 0.476 \\
\hline Brd & 2.81 & 0.57 & 2.86 & 0.61 & $2 \cdot 78$ & 0.60 & 2.76 & 0.62 & $2 \cdot 70$ & 0.57 & 2.76 & 0.59 & 2.76 & 0.56 & 2.71 & 0.55 & 2.72 & 0.55 & $2 \cdot 73$ & 0.56 & 2.76 & 0.60 & 2.77 & 0.59 & 2.80 & 0.59 & \\
\hline \multicolumn{28}{|c|}{ HDL-C (mmol/l) } \\
\hline Glu & 1.50 & 0.33 & 1.53 & 0.36 & 1.49 & 0.34 & 1.46 & 0.34 & 1.46 & 0.34 & 1.47 & 0.34 & 1.48 & 0.34 & 1.47 & 0.33 & 1.49 & 0.33 & 1.48 & 0.31 & 1.49 & 0.33 & 1.49 & 0.34 & 1.51 & 0.37 & 0.408 \\
\hline Brd & 1.47 & 0.31 & 1.52 & 0.35 & 1.48 & 0.32 & 1.47 & 0.31 & 1.44 & 0.31 & 1.46 & 0.34 & 1.47 & 0.34 & 1.45 & 0.32 & 1.45 & 0.31 & 1.45 & 0.32 & 1.46 & 0.31 & 1.47 & 0.33 & 1.48 & 0.32 & \\
\hline \multicolumn{28}{|c|}{ TAG (mmol/l) } \\
\hline Glu & 0.91 & 0.27 & 0.92 & 0.28 & 0.94 & 0.29 & 0.96 & 0.32 & 0.95 & 0.33 & 0.87 & 0.32 & $0.82 \dagger$ & 0.31 & 0.80 & 0.28 & 0.83 & 0.29 & 0.87 & 0.29 & 0.92 & 0.29 & 0.98 & 0.29 & $1 \cdot 01$ & 0.29 & 0.017 \\
\hline Brd & 0.88 & 0.27 & 0.89 & 0.27 & 0.92 & 0.24 & 0.94 & 0.27 & 0.93 & 0.30 & 0.93 & 0.33 & 0.91 & 0.31 & 0.89 & 0.32 & 0.89 & 0.30 & 0.90 & 0.30 & 0.93 & 0.32 & 0.95 & 0.33 & 0.98 & 0.33 & \\
\hline \multicolumn{28}{|c|}{ NEFA (mmol/l) } \\
\hline Glu & 0.47 & 0.19 & 0.45 & 0.20 & 0.35 & 0.16 & $0.22 \dagger$ & 0.10 & $0.15^{\star}$ & 0.05 & $0.10 \dagger$ & 0.03 & 0.10 & 0.03 & 0.16 & 0.07 & 0.32 & 0.15 & $0.49^{*}$ & 0.16 & $0.60^{*}$ & 0.18 & $0.69^{\star}$ & 0.22 & 0.71 & 0.20 & 0.001 \\
\hline Brd & 0.44 & 0.11 & 0.38 & 0.11 & 0.40 & 0.14 & 0.33 & 0.12 & 0.23 & 0.08 & 0.13 & 0.04 & 0.11 & 0.03 & 0.14 & 0.06 & 0.22 & 0.09 & 0.29 & 0.11 & 0.40 & 0.12 & 0.50 & 0.12 & 0.62 & 0.14 & \\
\hline
\end{tabular}

TC, total cholesterol; LDL-C, LDL-cholesterol; HDL-C, HDL-cholesterol

"Mean values were significantly different from those of the Brd challenge for each time point $(P<0.01)$.

† Mean values were significantly different from those of the Brd challenge for each time point $(P<0.05)$.

$\ddagger$ The values represent the absolute concentrations for each outcome. 

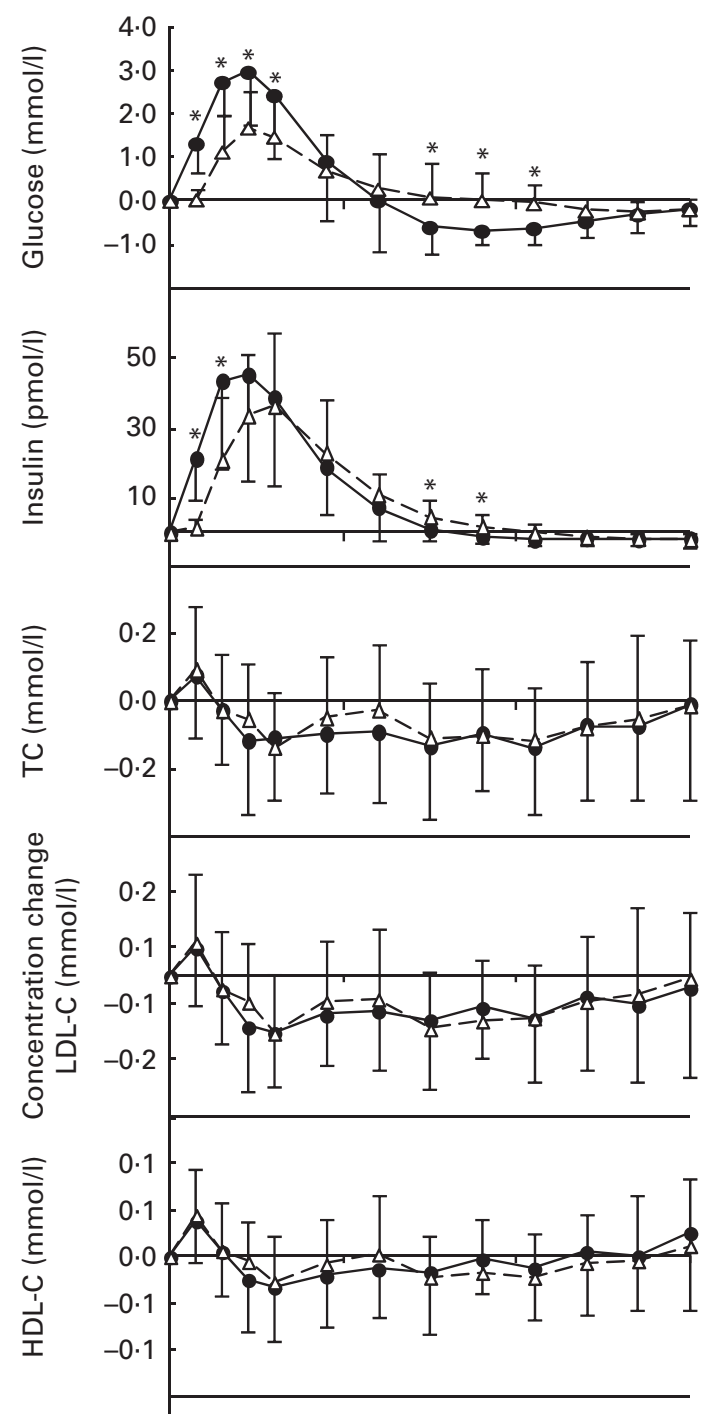
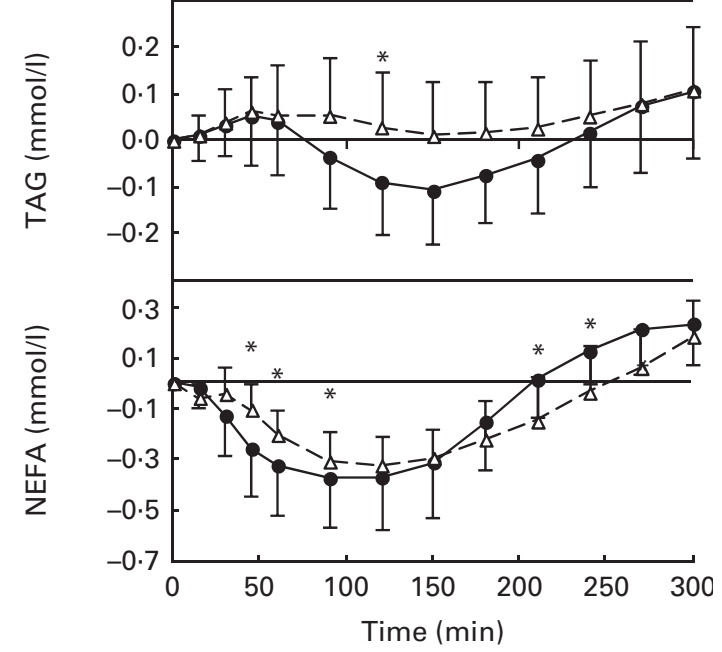

Fig. 1. Postprandial serum glucose, insulin, total cholesterol (TC), LDL-cholesterol (LDL-C), HDL-cholesterol (HDL-C), TAG and NEFA responses to the standard oral glucose $(\bullet,-)$ and white bread $\left(\triangle,-{ }_{-}\right)$challenges expressed as changes from the fasting concentrations. Values are means, with standard deviations represented by vertical bars. There was a significant effect for the treatment $\times$ time interaction: glucose, $P=0.001$; insulin, $P=0.0001$; TAG, $P=0.0165$; NEFA, $P=0.0006$. * Mean values were significantly different from those of the Brd challenge for each time point $(P<0.05)$.

and more recently for high- and low-glycaemic index meals that were ${ }^{(23)}$ or were not ${ }^{(24)}$ matched in macronutrient and fibre contents. Both glucose and white bread are used as standard reference foods for glycaemic index determinations. White bread elicits approximately $70 \%$ of the area under the glycaemic response curve as glucose does ${ }^{(18)}$. Of note were the differences between the two challenges after $2 \mathrm{~h}$ observed in the present study. Oral glucose elicited significantly lower serum glucose concentrations, in the absence of differences in insulin concentrations during the same time period. It has been reported previously in animal and human studies that feeding initiation occurs after a transient decline in blood glucose concentrations ${ }^{(25,26)}$. These data suggest that under free-living conditions subjects may be more likely to start eating earlier after orally consuming glucose than after consuming white bread, although we did not measure food cravings or subsequent food intake.

Both fasting and non-fasting TAG concentrations are independent risk factors for $\mathrm{CVD}^{(22)}$. Non-fasting $(2-4 \mathrm{~h}$ postprandial) compared with fasting TAG concentrations have been reported to be more strongly associated with cardiovascular events ${ }^{(27)}$. It is well established that high-carbohydrate diets are most closely associated with elevated TAG concentrations ${ }^{(9,28)}$. However, few studies have explored how different carbohydrates affect TAG metabolism in the postprandial state. Early intervention studies conducted in the 1970 s and 1980s had addressed this research question by assessing postprandial TAG responses to mixed meals after the participants consumed diets with different carbohydrate sources or amounts for $7-14 \mathrm{~d}^{(9,11,29)}$. The results from these studies consistently showed higher fasting TAG and a greater postprandial TAG response after consumption of diets with greater carbohydrate contents.

In contrast to previous studies, the present study evaluated the postprandial lipid responses to the acute intake of different carbohydrate sources. During the period when the TAG concentrations diverged for the two carbohydrate challenges, the oral glucose challenge resulted in lower concentrations than the white bread challenge. This finding was somewhat unexpected because we assumed that the more rapidly absorbable form of carbohydrate would promote a faster rate of chylomicron formation and subsequent release into the circulation, as has been documented previously using meals with different amounts of slowly available glucose in cereal products ${ }^{(30)}$. An alternate explanation is that the more rapid secretion of chylomicrons promoted TAG clearance via elevated lipoprotein lipase activity. Nevertheless, the maximum difference in postprandial TAG concentrations, observed at $150 \mathrm{~min}$, was small $(0.09 \mathrm{mmol} / \mathrm{l}$ or $80 \mathrm{mg} / \mathrm{l})$ and may not be meaningful clinically. Mean TAG concentrations observed in the present study remained below $2 \mathrm{mmol} / \mathrm{l}$ in the postchallenge period regardless of the test food consumed. This value has been recently suggested as a desirable value for non-fasting TAG concentrations when assessing cardiovascular risk in a clinical setting ${ }^{(22)}$. Further comparisons with clinical recommendations for postprandial TAG are implausible as the standard methodology for assessing postprandial 
lipaemia consists of conducting a fat tolerance test rather than administering a carbohydrate challenge.

Similar to the observation made in the present study, consumption of a low-glycaemic index meal elicited a greater postprandial TAG response than that of a high-glycaemic index meal in hypercholesterolaemic postmenopausal women $^{(24)}$ and in older adults (62-72 years) with or without impaired glucose tolerance ${ }^{(31)}$. The response of the other lipid parameters assessed in the present study, total cholesterol, LDL-cholesterol and HDL-cholesterol, was comparable for the two carbohydrate challenges.

Fasting serum NEFA concentrations have been related to carotid atherosclerotic plaque formation ${ }^{(32)}$ and markers of endothelial dysfunction ${ }^{(33)}$. Both carbohydrate challenges reduced serum NEFA concentrations from baseline concentrations, determined after a $12 \mathrm{~h}$ fast. This effect was more pronounced for the oral glucose than for the white bread challenge in the early phase of the observation period and for the white bread challenge more so than for the oral glucose challenge in the later phase of the observation period. These differences, although statistically significant, were modest. Previously, when postprandial NEFA were evaluated in the context of the glycaemic index, the drop in NEFA concentrations was not significantly different in response to mixed meals with high or low glycaemic indices ${ }^{(24,34)}$. Similar findings have been reported in response to long-term feeding of diets with different glycaemic indices ${ }^{(34,35)}$

A strength of the present study is that within the context of highly controlled environmental conditions, we assessed the effect of the acute intake of two common sources of dietary carbohydrates on postprandial serum glucose, insulin and lipids. The issue of a controlled environment is important given the potential effects of extraneous stimulation on the metabolic parameters assessed ${ }^{(36)}$. Additionally, duplicate determinations were made for each study participant.

Several limitations of the present study deserve consideration. Given the narrow range of foods tested, the present results cannot be extrapolated to foods or mixed meals with a broader range of macronutrient compositions. The observational period, although longer than that traditionally monitored, was limited to $5 \mathrm{~h}$. This period was chosen because it was estimated to be reasonable before a second meal occasion could be initiated. Neither food cravings nor subsequent food intake was assessed during the $5 \mathrm{~h}$ period to determine whether the changes observed might be related to the food intake behaviour. The possibility that the observed differences in responses to oral glucose and white bread may be attributed to factors not included as part of the present study cannot be ruled out. The two challenge carbohydrates, a solid and a liquid, were chosen because they are the two standard carbohydrates designated to be appropriate to determine glycaemic index values ${ }^{(37)}$. We cannot rule out the possibility that the different food matrices could have had a direct impact on the rate of intestinal absorption. However, an early study on the glycaemic and insulinaemic responses to drinks containing $50 \mathrm{~g}$ of glucose or soluble amylopectin has reported responses that were comparable with those observed in the present study ${ }^{(38)}$. It has been documented that adding different fats (approximately $30 \mathrm{~g}$ ) to white bread lowers its glycaemic response ${ }^{(39,40)}$, suggesting the possibility that the presence of lipids in the test foods (particularly bread) could have affected the postprandial responses studied herein. This issue was minimised by selecting a commercially available white bread with minimal amounts of dietary fat relative to the amount of available carbohydrate (3v $v$. 49\% by weight $)^{(41)}$. Finally, the amount of time given to the participants to consume the test foods ( $5 \mathrm{~min}$ ) may not reflect the usual time that individuals take to eat white bread, and may have slowed down starch digestion and subsequent glucose absorption. However, the aim of the present intervention was to mimic a bolus administration of the test foods precisely to minimise any variability that could be attributed to chewing time.

In conclusion, these data suggest that the type of carbohydrate used to determine the glycaemic index, bread or glucose, has modest, if any, effects on postprandial lipids. Further research should focus on potential second-meal effects and the chronic intake of foods containing a diverse range of carbohydrates on cardiometabolic disease risk and subsequent pathogenesis.

\section{Acknowledgements}

The authors acknowledge the study participants as well as the Metabolic Research Unit and Nutrition Evaluation Laboratory staff at the HNRC, Tufts University, for their cooperation. The present study was supported by the R01 DK073321 and a grant from the US Department of Agriculture to Tufts University, under agreement no. 58-1950-4-401. The authors contributions are as follows: S. V.-L., L. M. A. and A. H. L. designed the study and wrote the manuscript; S. V.-L. and A. H. L. conducted the study; S.V.-L., L. M. A., N. R. M. and A. H. L. conducted the data analysis. All the authors read and approved the final manuscript. None of the authors has conflicts of interest to disclose.

\section{References}

1. Livesey G, Taylor R, Hulshof T, et al. (2008) Glycemic response and health a systematic review and meta-analysis: relations between dietary glycemic properties and health outcomes. Am J Clin Nutr 87, 2585-2268.

2. Sheard NF, Clark NG, Brand-Miller JC, et al. (2004) Dietary carbohydrate (amount and type) in the prevention and management of diabetes. A statement by the American Diabetes Association. Diabetes Care 27, 2266-2271.

3. Jenkins DJA, Wolever TMS, Taylor RH, et al. (1981) Glycemic index of foods: a physiological basis for carbohydrate exchange. Am J Clin Nutr 34, 362-366.

4. Marsh K, Barclay AW, Colaquiri S, et al. (2011) Glycemic index and glycemic load of carbohydrates in the diabetes diet. Curr Diab Rep 11, 120-127.

5. Duffey KJ, Gordon-Larsen P, Steffen LM, et al. (2010) Drinking caloric beverages increases the risk of adverse cardiometabolic outcomes in the Coronary Artery Risk Development in Young Adults (CARDIA) Study. Am J Clin Nutr 92 954-959.

6. Howard BV \& Wylie-Rosett J (2002) Sugar and cardiovascular disease: a statement for healthcare professionals from the 
committee on nutrition of the council on Nutrition, Physical Activity and Metabolism of the American Heart Association. Circulation 106, 523-527.

7. Erkkila AT, Herrington DM, Mozaffarian D, et al. (2005) Cereal fiber and whole-grain intake are associated with reduced progression of coronary-artery atherosclerosis in postmenopausal women with coronary artery disease. $\mathrm{Am}$ Heart J 150, 94-101.

8. Welsh JA, Sharma A, Abramson JL, et al. (2010) Caloric sweetener consumption and dyslipidemia among US adults. JAMA 303, 1490-1497.

9. Coulston AM, Liu GC \& Reaven GM (1983) Plasma glucose, insulin and lipid responses to high-carbohydrate low-fat diets in normal humans. Metabolism 32, 52-56

10. Lichtenstein AH, Ausman LM, Carrasco W, et al. (1994) Shortterm consumption of a low-fat diet beneficially affects plasma lipid concentrations only when accompanied by weight loss. Hypercholesterolemia, low-fat diet, and plasma lipids. Arterioscler Thromb 14, 1751-1760.

11. Mann JI \& Truswell AS (1972) Effects of isocaloric exchange of dietary sucrose and starch on fasting serum lipids, postprandial insulin secretion and alimentary lipaemia in human subjects. Br J Nutr 27, 395-405.

12. Franz MJ (2008) Is there a role for the glycemic index in coronary heart disease prevention or treatment? Curr Atheroscler Rep 10, 497-502.

13. Vrolix R \& Mensink RP (2010) Effects of glycemic load on metabolic risk markers in subjects at increased risk of developing metabolic syndrome. Am J Clin Nutr 92, 366-374.

14. Crapo PA, Reaven GM \& Olefsky JM (1977) Postprandial plasma-glucose and -insulin responses to different complex carbohydrates. Diabetes 26, 1178-1183.

15. Reaven G (1979) Effects of differences in amount and kind of dietary carbohydrate on plasma glucose and insulin responses in man. Am J Clin Nutr 32, 2568-2578.

16. Wolever TMS, Yang M, Zeng XY, et al. (2006) Food glycemic index, as given in glycemic index tables, is a significant determinant of glycemic responses elicited by composite breakfast meals. Am J Clin Nutr 83, 1306-1312.

17. Brand-Miller JC, Thomas M, Swan V, et al. (2003) Physiological validation of the concept of glycemic load in lean young adults. J. Nutr 133, 2728-2732.

18. Wolever TMS, Jenkins DJA, Jenkins AL, et al. (1991) The glycemic index: methodology and clinical implications. $\mathrm{Am} \mathrm{J}$ Clin Nutr 54, 846-854.

19. Vega-López S, Ausman LM, Griffith JL, et al. (2007) Interindividual variability and intra-individual reproducibility of glycemic index values for commercial white bread. Diabetes Care 30, 1412-1417.

20. NCEP Expert Panel (2001) Executive summary of the third report of the National Cholesterol Education Program (NCEP) Expert Panel on Detection, Evaluation, and Treatment of High Blood Cholesterol in Adults (Adult Treatment Panel III). JAMA 285, 2486-2497.

21. Zilversmit DB (1979) Atherogenesis: a postprandial phenomenon. Circulation 60, 473-485.

22. Kolovou GD, Mikhailidis DP, Kovar J, et al. (2011) Assessment and clinical relevance of non-fasting and postprandial triglycerides: an Expert Panel statement. Curr Vasc Pharmacol 9, 258-270.

23. Krog-Mikkelsen I, Sloth B, Dimitrov D, et al. (2011) A low glycemic index diet does not affect postprandial energy metabolism but decreases postprandial insulinemia and increases fullness ratings in healthy women. J Nutr 141, $1679-1684$.
24. Bukkapatnam RN, Berglund L, Anuurad E, et al. (2010) Postprandial metabolic responses to dietary glycemic index in hypercholesterolemic postmenopausal women. Prev Cardiol 13, 29-35.

25. Smith FJ \& Campfield LA (1993) Meal initiation occurs after experimental induction of transient declines in blood glucose. Am J Physiol 265, R1423-R1429.

26. Campfield LA, Smith FJ, Rosenbaum M, et al. (1996) Human eating: evidence for a physiological basis using a modified paradigm. Neurosci Biobehav Rev 20, 133-137.

27. Bansal S, Buring JE, Rifai N, et al. (2007) Fasting compared with nonfasting triglycerides and risk of cardiovascular events in women. JAMA 298, 309-316.

28. Parks EJ (2001) Effect of dietary carbohydrate on triglyceride metabolism in humans. J Nutr 131, 2772S-22774.

29. Coulston AM, Hollenbeck CB, Swislocki ALM, et al. (1987) Deleterious metabolic effects of high-carbohydrate, sucrose-containing diets in patients with non-insulin-dependent diabetes mellitus. Am J Med 82, 213-220.

30. Harbis A, Perdreau S, Vincent-Baudry S, et al. (2004) Glycemic and insulinemic meal responses modulate postprandial hepatic and intestinal lipoprotein accumulation in obese, insulin-resistant subjects. Am J Clin Nutr 80, 896-902.

31. Perälä M-M, Hätönen KA, Virtamo J, et al. (2011) Impact of overweight and glucose tolerance on postprandial responses to high- and low-glycaemic index meals. Br J Nutr $\mathbf{1 0 5}$, 1627-1634.

32. Taniguchi A, Nakai Y, Fukushima M, et al. (2002) Ultrasonographically assessed carotid atherosclerosis in Japanese type 2 diabetic patients: role of nonesterified fatty acids. Metabolism 51, 539-543.

33. Mathew M, Tay E \& Cusi K (2010) Elevated plasma free fatty acids increase cardiovascular risk by inducing plasma biomarkers of endothelial activation, myeloperoxidase and PAI-1 in healthy subjects. Cardiovasc Diabetol 9, 9.

34. Brynes AE, Edwards CM, Ghatei MA, et al. (2003) A randomised four-intervention crossover study investigating the effect of carbohydrates on daytime profiles of insulin, glucose, non-esterified fatty acids and triacylglycerols in middleaged men. Br J Nutr 89, 207-218.

35. Wolever TMS \& Mehling CC (2003) Long-term effect of varying the source or amount of dietary carbohydrate on postprandial plasma glucose, insulin, triacylglycerol, and free fatty acid concentrations in subjects with impaired glucose tolerance. Am J Clin Nutr 77, 612-621.

36. Surwit R \& Schneider M (1993) Role of stress in the etiology and treatment of diabetes mellitus. Psychosom Med $\mathbf{5 5}$, 380-393.

37. Brouns F, Bjorck I, Frayn K, et al. (2005) Glycaemic index methodology. Nutr Res Rev 18, 145-171.

38. Crapo PA, Reaven GM \& Olefsky JM (1976) Plasma glucose and insulin responses to orally administered simple and complex carbohydrates. Diabetes 25, 741-747.

39. Jeya C, Henry K, Lightowler HJ, et al. (2008) The influence of adding fats of varying saturation on the glycaemic response of white bread. Int J Food Sci Nutr 59, 61-69.

40. MacIntosh CG, Holt SH \& Brand-Miller JC (2003) The degree of fat saturation does not alter glycemic, insulinemic or satiety responses to a starchy staple in healthy men. J Nutr $\mathbf{1 3 3}$, 2577-2580.

41. U.S. Department of Agriculture Agricultural Research Service (2012) USDA National Nutrient Database for Standard Reference, Release 25. Nutrient Data Laboratory. 